\title{
Analytic Feynman Integral and a Change of Scale Formula for Wiener Integrals of an Unbounded Cylinder Function
}

\author{
Kim Young Sik (D) \\ Department of Mathematics, College of Natural Sciences, Industry-University Cooperation Foundation, Hanyang University, \\ 222 Wangsimni-ro, Seongdong-gu, Seoul 04763, Republic of Korea
}

Correspondence should be addressed to Kim Young Sik; yoskim@hanyang.ac.kr

Received 18 June 2020; Revised 14 September 2020; Accepted 22 September 2020; Published 17 October 2020

Academic Editor: Dimitri Volchenkov

Copyright $\odot 2020$ Kim Young Sik. This is an open access article distributed under the Creative Commons Attribution License, which permits unrestricted use, distribution, and reproduction in any medium, provided the original work is properly cited.

We investigate the behavior of the unbounded cylinder function $F(x)=\left(\int_{0}^{T} \alpha_{1}(t) \mathrm{d} x(t)\right)^{2 k} \cdot\left(\int_{0}^{T} \alpha_{2}(t) \mathrm{d} x(t)\right)^{2 k} \ldots \ldots$ $\left(\int_{0}^{T} \alpha_{n}(t) \mathrm{d} x(t)\right)^{2 k}, k=1,2, \ldots$ whose analytic Wiener integral and analytic Feynman integral exist, we prove some relationships among the analytic Wiener integral, the analytic Feynman integral, and the Wiener integral, and we prove a change of scale formula for the Wiener integral about the unbounded function on the Wiener space $C_{0}[0, T]$.

\section{Introduction}

In [1], Cameron and Martin initially worked about the behavior of measure and measurability under change of scale in the Wiener space in 1947. In [2], Johnson and Skoug proved the scale-invariant measurability on the Wiener space in 1979. In [3, 4], Cameron and Storvick proved a change of scale formula for bounded functions on the Wiener space in 1987. In [5], Kim proved a change of scale formula for Wiener integrals about a function $F(x)=f\left(\left(h_{1}, x\right)^{\sim}, \ldots,\left(h_{n}, x\right)^{\sim}\right) \quad$ with $f \in L_{p}\left(R^{n}\right)$, $1 \leq p \leq \infty$ : the analytic Wiener integral exists for $f \in L_{p}\left(R^{n}\right), 1 \leq p \leq \infty$ and the analytic Feynman integral exists for $f \in L_{1}\left(R^{n}\right)$. In general, the analytic Feynman integral does not always exist for $f \in L_{p}\left(R^{n}\right)$ with $1<p$. In [6], Brue worked about the transform for Feynman integrals in 1972. In [7], Huffman et al. expanded the Fourier Feynman transform theory of $f\left(\int_{0}^{T} \alpha_{1} \mathrm{~d} x, \ldots, \int_{0}^{T} \alpha_{n} \mathrm{~d} x\right)$ for $f \in L_{p}\left(R^{n}\right)$ with $1 \leq p \leq 2$. In [8,9], Kim extended these results to the function $\widehat{\mu}\left(\left(h_{1} x\right)^{\sim}, \ldots,\left(h_{n}, x\right)^{\sim}\right)$, where $\widehat{\mu}$ is a Fourier transform of a complex-valued Borel measure $\mu$ in $\mathscr{M}\left(R^{n}\right)$, which is a space of complex-valued Borel measures. In [10], Kim investigated the behavior of a scale factor for Wiener integrals on the Wiener space.

In [11]-[13], Cameron and Martin expanded the theory about the translation and transformation theory for the
Wiener integral. In [14], Chung expanded the generalized integral transforms for Wiener integrals. In [15], Gaysinsky and Goldstein expanded the self-adjointness of Schrodinger operator and Wiener integrals. In [16], Johnson and Lapidus wrote the book about the Feynman integral and the Feynman's operational calculus. In [20], Kim proved the change of scale formula for Wiener integrals of cylinder functions of a Fourier transform of a measure.

In this paper, we investigate the behavior of a Wiener integral for the unbounded function $F(x)=\left(\int_{0}^{T} \alpha_{1}\right.$ $(t) \mathrm{d} x(t))^{2 k} \cdot\left(\int_{0}^{T} \alpha_{2}(t) \mathrm{d} x(t)\right)^{2 k} \cdots\left(\int_{0}^{T} \alpha_{n}(t) \mathrm{d} x(t)\right)^{2 k}, k=$ $1,2, \ldots$ and we prove that $F(x)$ is Wiener integrable and the analytic Wiener integral and the analytic Feynman integral of $F(x)$ exist. We also prove some relationships among the analytic Wiener integral, the analytic Feynman integral, and the Wiener integral and prove a change of scale formula for the Wiener integral of the unbounded function $F(x)$ on the Wiener space $C_{0}[0, T]$.

\section{Definitions and Preliminaries}

Throughout this paper, let $\mathbf{R}^{n}$ denote the $n$-dimensional Euclidean space and let $\mathbf{C}, \mathbf{C}_{+}$, and $\mathbf{C}_{+}^{\sim}$ denote the set of complex numbers, the set of complex numbers with positive real part, and the set of nonzero complex numbers with nonnegative real part, respectively. 
Let $C_{0}[0, T]$ denote the space of real-valued continuous functions $x$ on $[0, T]$ such that $x(0)=0$. Let $\mathscr{M}$ denote the class of all Wiener measurable subsets of $C_{0}[0, T]$, let $m$ denote a Wiener measure, and let $\left(C_{0}[0, T], \mathscr{M}, m\right)$ be a Wiener measure space; we denote the Wiener integral of a function $F: C_{0}[0, T] \longrightarrow$ C by $\int_{C_{0}[0, T]} F(x) \mathrm{d} m(x)$.

A subset $E$ of $C_{0}[0, T]$ is said to be scale-invariant measurable if $\rho E \in \mathscr{M}$ for each $\rho>0$, and a scale-invariant measurable set $N$ is said to be scale-invariant null if $m(\rho N)=0$ for each $\rho>0$. A property that holds except on a scale-invariant null set is said to hold scale-invariant almost everywhere (sa.e.). If two functionals $F$ and $G$ are equal s-a.e., we write $F \approx G$.

Definition 1. Let $F$ be a complex-valued measurable function on $C_{0}[0, T]$ such that the integral

$$
J(F ; \lambda)=\int_{C_{0}[0, T]} F\left(\lambda^{-(1 / 2)} x\right) \mathrm{d} m(x)
$$

exists for all real $\lambda>0$. If there exists a function $J^{*}(F ; z)$ analytic on $\mathbf{C}_{+}$such that $J^{*}(F ; \lambda)=J(F ; \lambda)$ for all real $\lambda>0$, then we define $J^{*}(F ; z)$ to be the analytic Wiener integral of $F$ over $C_{0}[0, T]$ with parameter $z$, and for each $z \in \mathbf{C}_{+}$, we write

$$
I^{\mathrm{aw}}(F ; z)=J^{*}(F ; z) \equiv \int_{C_{0}[0, T]}^{\mathrm{anw}_{z}} F(x) \mathrm{d} m(x) .
$$

Let $q$ be a nonzero real number and let $F$ be a function defined on $C_{0}[0, T]$ whose analytic Wiener integral exists for each $z$ in $\mathbf{C}_{+}$. If the following limit exists, then we call it the analytic Feynman integral of $F$ over $C_{0}[0, T]$ with parameter $q$, and we write

$$
I^{\mathrm{af}}(F ; q)=\lim _{z \longrightarrow-i q} I^{\mathrm{aw}}(F ; z) \equiv \int_{C_{0}[0, T]}^{\mathrm{anw}_{q}} F(x) \mathrm{d} m(x),
$$

where $z$ approaches $-i q$ through $\mathbf{C}_{+}$and $i^{2}=-1$.

Theorem 1. Wiener integration formula:

$$
\begin{gathered}
\int_{C_{0}[0, T]} f\left(\int_{0}^{T} \alpha_{1}(t) \mathrm{d} x(t), \ldots, \int_{0}^{T} \alpha_{n}(t) \mathrm{d} x(t)\right) \mathrm{d} m(x) \\
=\left(\frac{1}{2 \pi}\right)^{(n / 2)} \int_{\mathbf{R}^{n}} f(\vec{u}) \exp \left\{-\frac{1}{2} \sum_{j=1}^{n} u_{j}^{2}\right\} \mathrm{d} \vec{u},
\end{gathered}
$$

where $\left\{\alpha_{1}(t), \alpha_{2}(t), \ldots, \alpha_{n}(t)\right\}$ is an orthonormal set of elements in $L_{2}[0, T], f: \mathbf{R}^{n} \longrightarrow \mathbf{C}$ is a Lebesgue measurable function, $\vec{u}=\left(u_{1}, u_{2}, \ldots, u_{n}\right)$, and $d \vec{u}=d u_{1}, d u_{2}, \ldots$ , $d u_{n}$.

Remark 1. We will use several times the following wellknown integration formula:

$$
\int_{\mathbf{R}} \exp \left\{-a u^{2}+i b u\right\} \mathrm{d} u=\sqrt{\frac{\pi}{a}} \exp \left\{-\frac{b^{2}}{4 a}\right\},
$$

where $a$ is a complex number with $\operatorname{Re} a>0$ and $b$ is a real number.

\section{Main Results}

Define the unbounded function $\quad F(x)=f\left(\int_{0}^{T} \alpha_{1}\right.$
$\left.(t) \mathrm{d} x(t), \ldots, \int_{0}^{T} \alpha_{n}(t) \mathrm{d} x(t)\right)$ :

$$
\begin{aligned}
F(x)= & \left(\int_{0}^{T} \alpha_{1}(t) \mathrm{d} x(t)\right)^{2 k} \cdot\left(\int_{0}^{T} \alpha_{2}(t) \mathrm{d} x(t)\right)^{2 k} \cdots \\
& \cdot\left(\int_{0}^{T} \alpha_{n}(t) \mathrm{d} x(t)\right)^{2 k}, \quad k=1,2, \ldots
\end{aligned}
$$

where $\left\{\alpha_{1}(t), \alpha_{2}(t), \ldots, \alpha_{n}(t)\right\}$ is an orthonormal set in $L_{2}[0, T]$ and $f(\vec{u})=u_{1}^{2 k} u_{2}^{2 k}, \ldots, u_{n}^{2 k} \notin L_{p}\left(R^{n}\right), \quad 1 \leq p \leq \infty$ and $f(\vec{u})$ is unbounded.

To expand main results of this paper, we prove some lemmas.

Lemma 1. For $k=1,2, \ldots$ and for $z \in \mathbf{C}^{+}$, we have that

$$
\int_{-\infty}^{+\infty} u^{2 k} \exp \left\{-\frac{1}{2 z} u^{2}\right\} \mathrm{d} u=\sqrt{\frac{2 \pi}{z}} \cdot \frac{(2 k) !}{k !} \cdot\left(\frac{1}{2 z}\right)^{k} .
$$

Proof. First we know that for $z \in \mathbf{C}^{+}$and for $v \in \mathbf{R}$,

$$
\int_{-\infty}^{+\infty} \exp \left\{-\frac{z}{2} x^{2}+v x\right\} \mathrm{d} x=\sqrt{\frac{2 \pi}{z}} \cdot \exp \left\{\frac{v^{2}}{2 z}\right\} .
$$

Using the series expansion of the exponential function $e^{z}=\sum_{n=0}^{\infty}(1 / n !) z^{n}$ with $z \in \mathbf{C}$, we have that for $z \in \mathbf{C}^{+}$and for $v \in \mathbf{R}$, 


$$
\begin{aligned}
\int_{-\infty}^{+\infty} \exp \left\{-\frac{z}{2} x^{2}+v x\right\} \mathrm{d} x \\
\quad=\int_{-\infty}^{+\infty} \exp \left\{-\frac{z}{2} x^{2}\right\} \cdot\left(1+(v x)+\frac{1}{2 !}(v x)^{2}+\cdots+\frac{1}{k !}(v x)^{k}+\cdots\right) \mathrm{d} x \\
\quad=\int_{-\infty}^{+\infty} \exp \left\{-\frac{z}{2} x^{2}\right\}+(v x) \cdot \exp \left\{-\frac{z}{2} x^{2}\right\}+\frac{1}{2 !}(v x)^{2} \cdot \exp \left\{-\frac{z}{2} x^{2}\right\}+\cdots \cdots+\frac{1}{(2 k) !}(v x)^{k} \cdot \exp \left\{-\frac{z}{2} x^{2}\right\}+\cdots \mathrm{d} x \\
=\int_{-\infty}^{+\infty} \exp \left\{-\frac{z}{2} x^{2}\right\}+\frac{1}{2 !}(v x)^{2} \cdot \exp \left\{-\frac{z}{2} x^{2}\right\}+\cdots \cdots+\frac{1}{(2 k) !}(v x)^{2 k} \cdot \exp \left\{-\frac{z}{2} x^{2}\right\}+\cdots \mathrm{d} x \\
=\int_{-\infty}^{+\infty} \sum_{k=0}^{\infty} \frac{1}{(2 k) !} v^{2 k} \cdot u^{2 k} \cdot e^{-(z / 2) u^{2}} \mathrm{~d} u \\
=\sum_{k=0}^{\infty} \frac{1}{(2 k) !} v^{2 k} \cdot \int_{-\infty}^{+\infty} u^{2 k} \cdot e^{-(z / 2) u^{2}} \mathrm{~d} u .
\end{aligned}
$$

And for $z \in \mathbf{C}^{+}$,

$$
\sqrt{\frac{2 \pi}{z}} \exp \left\{\frac{v^{2}}{2 z}\right\}=\sqrt{\frac{2 \pi}{z}} \sum_{k=0}^{\infty} \frac{1}{k !}\left(\frac{v^{2}}{2 z}\right)^{k} .
$$

By (8)-(10), we have the desired result.
Lemma 2. Let $F: C_{0}[0, T] \longrightarrow \mathbf{C}$ be the unbounded function defined by (6). Then, for $k=1,2, \ldots, F(x)$ is a Wiener integrable function of $x \in C_{0}[0, T]$ and the Wiener integral is

$$
\int_{C_{0}[0, T]} F(x) \mathrm{d} m(x)=\left(\frac{(2 k) !}{k ! 2^{k}}\right)^{n} .
$$

Proof. By (4) and by Lemma 1, we have that

$$
\begin{aligned}
& \int_{C_{0}[0, T]} F(x) \mathrm{d} m(x) \\
& =\left(\frac{1}{2 \pi}\right)^{(n / 2)} \cdot \int_{R^{n}} u_{1}^{2 k} \cdot u_{2}^{2 k} \cdots \cdots u_{n}^{2 k} \cdot \exp \left\{-\frac{1}{2} \sum_{j=1}^{n} u_{j}^{2}\right\} \mathrm{d} \vec{u} \\
& =\left(\frac{1}{2 \pi}\right)^{(n / 2)}\left[\sqrt{2 \pi} \cdot \frac{(2 k) !}{k !} \cdot\left(\frac{1}{2}\right)^{k}\right]^{n} \\
& =\left(\frac{(2 k) !}{k ! 2^{k}}\right)^{n} .
\end{aligned}
$$

Remark 2. By Lemma 2, we have interesting Wiener integrals about the unbounded function: for an orthonormal set $\left\{\alpha_{1}, \alpha_{2}, \ldots, \alpha_{n}\right\}$ in $L_{2}[0, T]$,

$$
\begin{aligned}
& \int_{C_{0}[0, T]}\left(\int_{0}^{T} \alpha_{1}(t) \mathrm{d} x(t)\right)^{2} \cdot\left(\int_{0}^{T} \alpha_{2}(t) \mathrm{d} x(t)\right)^{2} \cdots\left(\int_{0}^{T} \alpha_{n}(t) \mathrm{d} x(t)\right)^{2} \mathrm{~d} m(x)=1, \\
& \int_{C_{0}[0, T]}\left(\int_{0}^{T} \alpha_{1}(t) \mathrm{d} x(t)\right)^{4} \cdot\left(\int_{0}^{T} \alpha_{2}(t) \mathrm{d} x(t)\right)^{4} \cdots\left(\int_{0}^{T} \alpha_{n}(t) \mathrm{d} x(t)\right)^{4} \mathrm{~d} m(x)=3^{n}, \\
& \int_{C_{0}[0, T]}\left(\int_{0}^{T} \alpha_{1}(t) \mathrm{d} x(t)\right)^{6} \cdot\left(\int_{0}^{T} \alpha_{2}(t) \mathrm{d} x(t)\right)^{6} \cdots\left(\int_{0}^{T} \alpha_{n}(t) \mathrm{d} x(t)\right)^{6} \mathrm{~d} m(x)=15^{n},
\end{aligned}
$$


Because $F(x)$ is a Wiener integrable function even though it is unbounded, we can challenge to prove the change of scale formula for the Wiener integral about the unbounded function $F(x)$ in (6) on the Wiener space $C_{0}[0, T]$.

First, we prove the existence of the analytic Wiener integral and the analytic Feynman integral about the unbounded function $F(x)$ in (6) on the Wiener space $C_{0}[0, T]$.

Theorem 2. Let $F: C_{0}[0, T] \longrightarrow \mathbf{C}$ be the unbounded function defined by (6). Then, for $z \in \mathbf{C}^{+}$and for $k=1,2, \ldots$, the analytic Wiener integral and the analytic Feynman integral of $F(x)$ exist and are given by

$$
\begin{aligned}
& \text { (1) } \int_{C_{0}[0, T]}^{\mathrm{anw}_{z}} F(x) \mathrm{d} m(x)=\left(\frac{(2 k) !}{k ! \cdot z^{k} \cdot 2^{k}}\right)^{n}, \\
& \text { (2) } \int_{C_{0}[0, T]}^{\mathrm{anf}_{q}} F(x) \mathrm{d} m(x)=\left(\frac{(2 k) !}{k ! \cdot(-i q)^{k} \cdot 2^{k}}\right)^{n},
\end{aligned}
$$

whenever $z \longrightarrow-$ iq through $\mathbf{C}^{+}$.

Proof. By (4) and by Lemma 2, we have that for real $z>0$ and for $k=1,2, \ldots$,

$$
\begin{aligned}
& \int_{C_{0}[0, T]} F\left(z^{-(1 / 2)} x\right) \mathrm{d} m(x) \\
& =\int_{C_{0}[0, T]}\left(z^{-(1 / 2)} \int_{0}^{T} \alpha_{1}(t) \mathrm{d} x(t)\right)^{2 k} \cdot\left(z^{-(1 / 2)} \int_{0}^{T} \alpha_{2}(t) \mathrm{d} x(t)\right)^{2 k} \cdots\left(z^{-(1 / 2)} \int_{0}^{T} \alpha_{n}(t) \mathrm{d} x(t)\right)^{2 k} \mathrm{~d} m(x) \\
& =z^{-\mathrm{kn}} \int_{C_{0}[0, T]}\left(\int_{0}^{T} \alpha_{1}(t) \mathrm{d} x(t)\right)^{2 k} \cdot\left(\int_{0}^{T} \alpha_{2}(t) \mathrm{d} x(t)\right)^{2 k} \cdots\left(\int_{0}^{T} \alpha_{n}(t) \mathrm{d} x(t)\right)^{2 k} \mathrm{~d} m(x) \\
& =z^{-\mathrm{kn}}\left(\frac{(2 k) !}{k ! \cdot 2^{k}}\right)^{n} \\
& =\left(\frac{(2 k) !}{k ! \cdot z^{k} \cdot 2^{k}}\right)^{n}
\end{aligned}
$$

By the analytic continuation of $z \in \mathbf{C}^{+}$, we can deduce the desired analytic Wiener integral and the analytic Feynman integral of $F(x)$ on the Wiener space $C_{0}[0, T]$

Remark 3. In Theorem 2, we prove that the analytic Wiener integral and the analytic Feynman integral about the unbounded function $F$ can exist, even though $f \notin L_{p}\left(R^{n}\right)$, $1 \leq p \leq \infty$, on the Wiener space $C_{0}[0, T]$.

To investigate the behavior of a change of scale formula for the Wiener integral, we prove some relationships between the Wiener integral and the analytic Wiener integral about the unbounded function $F$ in (6) on the Wiener space $C_{0}[0, T]$.
Lemma 3. Let $F: C_{0}[0, T] \longrightarrow \mathbf{C}$ be the unbounded function defined by (6). For $z \in \mathbf{C}^{+}$,

$$
\exp \left\{\frac{1-z}{2} \sum_{j=1}^{n}\left(\int_{0}^{T} \alpha_{j}(t) \mathrm{d} x(t)\right)^{2}\right\} F(x),
$$

is a Wiener integrable function of $x \in C_{0}[0, T]$.

Proof. By (4) and by (7), we have that for $z \in \mathbf{C}^{+}$and for $k=1,2, \ldots$, 


$$
\begin{aligned}
& \int_{C_{0}[0, T]} \exp \left\{\frac{1-z}{2} \sum_{j=1}^{n}\left(\int_{0}^{T} \alpha_{j}(t) \mathrm{d} x(t)\right)^{2}\right\} F(x) \mathrm{d} m(x) \\
& \quad=\left(\frac{1}{2 \pi}\right)^{(n / 2)} \int_{R^{n}} \exp \left\{\frac{1-z}{2} \sum_{j=1}^{n} u_{j}^{2}\right\} \cdot u_{1}^{2 k} \cdot u_{2}^{2 k} \cdots \cdots u_{n}^{2 k} \cdot \exp \left\{-\frac{1}{2} \sum_{j=1}^{n} u_{j}^{2}\right\} \mathrm{d} \vec{u} \\
& =\left(\frac{1}{2 \pi}\right)^{(n / 2)} \int_{R^{n}} u_{1}^{2 k} \cdot u_{2}^{2 k} \cdots \cdots u_{n}^{2 k} \cdot \exp \left\{-\frac{z}{2} \sum_{j=1}^{n} u_{j}^{2}\right\} \mathrm{d} \vec{u} \\
& =\left(\frac{1}{2 \pi}\right)^{(n / 2)} \cdot\left(\frac{2 \pi}{z}\right)^{(n / 2)} \cdot\left(\frac{(2 k) !}{k ! \cdot 2^{k} \cdot z^{k}}\right)^{n} \\
& =z^{-(n / 2)} \cdot\left(\frac{(2 k) !}{k ! \cdot 2^{k} \cdot z^{k}}\right)^{n} \\
& <\infty .
\end{aligned}
$$

Therefore, we have the desired result.

We prove the relationship between the analytic Wiener integral and the Wiener integral for the unbounded function $F(x)$ in (6). That is, we prove that the analytic Wiener integral of $F(x)$ can be successfully expressed as the sequence of Wiener integrals on the Wiener space $C_{0}[0, T]$.

Theorem 3. Let $F: C_{0}[0, T] \longrightarrow \mathbf{C}$ be the unbounded function defined by (6). Then, for $z \in \mathbf{C}^{+}$, the analytic Wiener integral of $F(x)$ can be successfully expressed as the sequence of Wiener integrals:

$$
\begin{aligned}
& \int_{C_{0}[0, T]}^{\mathrm{ann} w_{z}} F(x) \mathrm{d} m(x) \\
& \quad=z^{(n / 2)} \int_{C_{0}[0, T]} \exp \left\{\frac{1-z}{2} \sum_{j=1}^{n}\left(\int_{0}^{T} \alpha_{j}(t) \mathrm{d} x(t)\right)^{2}\right\} F(x) \mathrm{d} m(x) .
\end{aligned}
$$

Proof. By the proof of Lemma 3, we have that for $z \in \mathbf{C}^{+}$and for $k=1,2, \ldots$,

$$
\begin{aligned}
& \int_{C_{0}[0, T]} \exp \left\{\frac{1-z}{2} \sum_{j=1}^{n}\left(\int_{0}^{T} \alpha_{j}(t) \mathrm{d} x(t)\right)^{2}\right\} F(x) \mathrm{d} m(x) \\
& =z^{-(n / 2)} \cdot\left(\frac{(2 k) !}{k ! \cdot 2^{k} \cdot z^{k}}\right)^{n} \\
& =z^{-(n / 2)} \int_{C_{0}[0, T]}^{a n w_{z}} F(x) \mathrm{d} m(x) .
\end{aligned}
$$

We prove that the unbounded function $F(x)$ in (6) successfully satisfies the change of scale formula for the Wiener integral on the Wiener space $C_{0}[0, T]$.

Theorem 4. Let $F: C_{0}[0, T] \longrightarrow \mathbf{C}$ be the unbounded function defined by (6). Then, for a positive real $\rho>0$,

$$
\begin{aligned}
& \int_{C_{0}[0, T]} F(\rho x) \mathrm{d} m(x) \\
& \quad=\rho^{-n} \int_{C_{0}[0, T]} \exp \left\{\frac{\rho^{2}-1}{2 \rho^{2}} \sum_{j=1}^{n}\left(\int_{0}^{T} \alpha_{j}(t) \mathrm{d} x(t)\right)^{2}\right\} F(x) \mathrm{d} m(x) .
\end{aligned}
$$

Proof. By Theorem 3, we have that for real $z>0$,

$$
\begin{aligned}
& \int_{C_{0}[0, T]} F\left(z^{-(1 / 2)} x\right) \mathrm{d} m(x) \\
& =z^{(n / 2)} \int_{C_{0}[0, T]} \exp \left\{\frac{1-z}{2} \sum_{j=1}^{n}\left(\int_{0}^{T} \alpha_{j}(t) \mathrm{d} x(t)\right)^{2}\right\} F(x) \mathrm{d} m(x) .
\end{aligned}
$$

If we let $z=\rho^{-2}$ in the above equation, we have the desired result.

Finally, we prove the relationship between the analytic Feynman integral and the Wiener integral. That is, we prove that the analytic Feynman integral of the unbounded function $F(x)$ can be successfully expressed as the limit of the sequence of Wiener integrals on the Wiener space $C_{0}[0, T]$.

Theorem 5. Let $F: C_{0}[0, T] \longrightarrow \mathbf{C}$ be the unbounded function defined by (6). Then, the analytic Feynman integral 
of $F(x)$ can be successfully expressed as the limit of the sequence of analytic Wiener integrals:

$$
\int_{C_{0}[0, T]}^{\operatorname{anf}_{q}} F(x) \mathrm{d} m(x)=\lim _{s \longrightarrow \infty}(n / 2)_{s}^{(n / 2)} \int_{C_{0}[0, T]} \exp \left\{\frac{1-z_{s}}{2} \sum_{j=1}^{n}\left(\int_{0}^{T} \alpha_{j}(t) \mathrm{d} x(t)\right)^{2}\right\} F(x) \mathrm{d} m(x),
$$

whenever $\left\{z_{s}\right\}_{s \in N} \longrightarrow-$ iq through $\mathbf{C}_{+}$with $N=\{1,2,3, \ldots\}$.

$$
\begin{aligned}
\int_{C_{0}[0, T]}^{\operatorname{anf} f_{q}} F(x) \mathrm{d} m(x) & =\lim _{z_{s} \longrightarrow-i q} \int_{C_{0}[0, T]}^{\mathrm{anw}_{z_{s}}} F(x) \mathrm{d} m(x) \\
& =\lim _{s \longrightarrow \infty}(n / 2)_{s}^{(n / 2)} \int_{C_{0}[0, T]} \exp \left\{\frac{1-z_{s}}{2} \sum_{j=1}^{n}\left(\int_{0}^{T} \alpha_{j}(t) \mathrm{d} x(t)\right)^{2}\right\} F(x) \mathrm{d} m(x),
\end{aligned}
$$

Proof. By Theorem 3 and by the definition of the analytic Feynman integral, we have that whenever $\left\{z_{s}\right\}_{s \in N} \longrightarrow-i q$ through $\mathbf{C}_{+}$.

Remark 4. The motivation of this paper follows from the notation $f(x)=\left\langle x, \alpha_{1}>^{n_{1}}, \ldots,<x, \alpha_{r}>^{n_{r}}\right.$ and by some properties on the Hilbert space in $[18,19]$. To check the existence of the analytic Wiener integral and the analytic Feynman integral of $F(x)$, we take $n_{1}=n_{2}=\cdots=n_{r}=2 k$ and there are no other reasons about this choice.

\section{Data Availability}

The data used to support the findings of this study are included within this article.

\section{Disclosure}

The abstract of this paper was presented in the international conference of Korean Mathematical Society in 2017 [20].

\section{Conflicts of Interest}

The author declares that there are no conflicts of interest regarding the publication of this paper.

\section{Acknowledgments}

This study was supported by the National Research Foundation of Korea (grant no. NRF-2017R1A6A3A11030667).

\section{References}

[1] R. H. Cameron and W. T. Martin, "The behavior of measure and measurability under change of scale in Wiener space," Bulletin of the American Mathematical Society, vol. 53, pp. 130-137, 1947.

[2] G. W. Johnson and D. L. Skoug, "Scale-invariant measurablity in Wiener space," Pacific Journal of Mathematics, vol. 283, pp. 157-176, 1979.
[3] R. H. Cameron and D. A. Storvick, "Relationships between the Wiener integral and the analytic Feynman integral," Supplemento ai Rendiconti del Circolo Matematico di Palermo, Serie II-Numero, vol. 17, pp. 117-133, 1987.

[4] R. H. Cameron and D. A. Storvick, "Change of scale formulas for Wiener integral," Supplemento ai Rendiconti del Circolo Matematico di Palermo, Serie II-Numero, vol. 17, pp. 105-115, 1987.

[5] Y. S. Kim, "A change of scale formula for Wiener integrals of cylinder functions on the abstract Wiener space," International Journal of Mathematics and Mathematical Sciences, vol. 21, no. 1, pp. 73-78, 1998.

[6] M. D. Brue, "A functional transform for feynman integrals similar to fourier transform," Thesis, University of Minnesota, Minneapolis, MN, USA, 1972.

[7] T. Huffman, C. Park, and D. Skoug, "Analytic fourier Feynman transforms and convolutions," Transactions of the American Mathematical Society, vol. 347, no. 2, pp. 661-673, 1995.

[8] Y.S. Kim, "Fourier Feynman transform and analytic Feynman integrals and convolutions of a fourier transform of a measure on Wiener spaces," Houston Journal of Mathematics, vol. 36, no. 1, pp. 1139-1158, 2010.

[9] Y. S. Kim, "Behavior of the first variation of Fourier transform of a measure on the Fourier Feynman transform and convolution," Numerical Functional Analysis and Optimization, vol. 37, no. 6, pp. 699-718, 2016.

[10] Y.S. Kim, "Behavior of a scale factor for Wiener integrals and a fourier Stieltjes transform on the Wiener space," Applied Mathematics, vol. 9, no. 5, pp. 488-495, 2018.

[11] R. H. Cameron, "The translation pathology of Wiener space," Duke Mathematical Journal, vol. 21, no. 4, pp. 623-628, 1954.

[12] R. H. Cameron and W. T. Martin, "On transformations of Wiener integrals under translations," Annals of Mathematics, vol. 45, no. 2, pp. 386-396, 1944.

[13] R. H. Cameron and W. T. Martin, "Transformations for Wiener integrals under a general class of linear transformations," Transactions of the American Mathematical Society, vol. 58, no. 2, pp. 184-219, 1945. 
[14] H. S. Chung, "Generalized integral transorms via the series expressions," Mathematics, vol. 8, no. 4, p. 539, 2020.

[15] M. D. Gaysinsky and M. S. Goldstein, "Self-adjointness of Schrödinger operator and Wiener integrals," Integral Equations and Operator Theory, vol. 15, pp. 973-990, 1992.

[16] G. W. Johnson and M. L. Lapidus, The Feynman Integral and Feynman's Operational Calculus, Oxford Science Publications, Oxford, UK, 2000.

[17] Y. S. Kim, "A change of scale formula for Wiener integrals of cylinder functions on the abstract Wiener space II," International Journal of Mathematics and Mathematical Sciences, vol. 25, no. 4, 7 pages, Article ID 804515, 2001.

[18] N. Hayek, B. J. González, and E. R. Negrin, "Matrix Wiener transform," Applied Mathematics and Computation, vol. 218, no. 3, pp. 773-776, 2011.

[19] H. Hayek, S. M. Srivastava, B. J. Gonzalez, and E. R. Negrin, "A family of Wiener transforms associated with a pair of operators on Hilbert space," Integral Transforms and Special Functions, vol. 24, no. 1, pp. 1-8, 2013.

[20] Y. S. Kim, "Behavior of a change of scale for Wiener integrals of unbounded functions," Conference of Korean Mathematical Society, vol. 2017, 2017 no. 0097. 